\title{
Which Gloves Are Efficient To Protect Against Titanium Dioxide Nanoparticles In Work Conditions?
}

\author{
Ludwig Vinches*, Stéphane Hallé1, Caroline Peyrot, Kevin J. Wilkinson² \\ 1 École de technologie supérieure \\ 1100 Notre-Dame Ouest, Montreal (QC) H3C 1K3 Canada \\ ${ }^{2}$ Dept. of Chemistry, University of Montreal \\ C.P. 6128, succ. Centre-ville, Montreal (QC) H3C 3J7 Canada
}

\begin{abstract}
Recent articles underline the potential health risks associated to the "nano" revolution. Titanium dioxide nanoparticles $\left(\mathrm{nTiO}_{2}\right)$ are one of these engineered nanoparticles (ENP) that have been cautioned about their likely harmful effects on health. In occupational use, to handle ENP, many Health \& Safety agencies have recommended the application of the precautionary principle namely the recommendation of the use of protective gloves against chemicals. However, at the best of our knowledge, no study about the penetration of ENP through protective gloves in working conditions was performed. This study was designed to evaluate the efficiency of several models of protective gloves against $\mathrm{nTiO}_{2}$. Two types of nitrile rubber gloves (100 $\mu \mathrm{m}$ and $200 \mu \mathrm{m})$, latex and butyl rubber gloves were brought into contact with $\mathrm{nTiO}_{2}$ in water, in propylene glycol (PG) or in powder. Mechanical biaxial deformations (BD), simulating the flexing of the hand, were applied to the samples during their exposure to ENP.

Depending the model of gloves and the mode of application of the NP, the results obtained by ICP-MS (Inductively Coupled Plasma - Mass Spectrometry) are different. For nTiO 2 in water, the passage is highlighted for nitrile rubber gloves $(100 \mu \mathrm{m})$ after only 60 deformations and the $\mathrm{nTiO}_{2}$ concentration reaches its maximum for $180 \mathrm{BD}$. Regarding the $\mathrm{nTiO}_{2}$ in powder, nitrile rubber gloves $(100 \mu \mathrm{m})$ and butyl rubber, the values achieved are significant but less than the solutions.
\end{abstract}

Keywords: $\mathrm{TiO}_{2}$ nanoparticles, Protective gloves, Work conditions, Glove efficiency, ICP-MS

(C) Copyright 2014 Authors - This is an Open Access article published under the Creative Commons Attribution License terms (http://creativecommons.org/licenses/by/3.0). Unrestricted use, distribution, and reproduction in any medium are permitted, provided the original work is properly cited.

${ }^{*}$ Corresponding author: ludwig.vinches.1@ens.etsmtl.ca

\section{Introduction}

The increasing use of ENP in commercial products and industrial processes make their occupational exposures inevitable $[1,2]$. Indeed, the global market for nanotechnologies will attain over 2.5 trillion dollars in 2020 [3] and at the same time, the workers and the researchers in this industry will reach 6 million persons [4]. Concerning $\mathrm{nTiO}_{2}$, they are increasingly present in several commercial products like paints, varnishes, sunscreens, etc. [5, 6]. But recently, the International Agency for Research on Cancer (IARC) has classified nanosized titanium dioxide in 2B-group as possibly carcinogenic to humans [7]. This decision follows numerous studies caution about their likely harmful effects on health. For example, a small increase in the number of cancer among workers in contact with $\mathrm{nTiO}_{2}$ has been reported [8]. Moreover, study conducted on hairless mice and porcine skin after subchronic dermal exposure to $\mathrm{nTiO}_{2}$ have shown that the ENP can be located in deep layer of epidermis [9]. After 60 days dermal exposure, $\mathrm{nTiO}_{2}$ reach different tissues and pathological lesions can be observed. Others studies indicated the penetration of ENP through intact or damaged human skin [10-12].

In response to IARC's classification, several Health \& Safety government agencies have recommended the application of the precautionary principle $[13,14]$ like the use of protective gloves against chemicals even if no thorough scientific validation of their efficiency against nanoparticles has been made yet.

At the best of our knowledge, three groups have reported research carried out on protective gloves against nanoparticles but most of it involves aerosols. In 2008, Golanski et al has reported the diffusion of 30 and $80 \mathrm{~nm}$ graphite nanoparticles through nitrile, vinyl, latex and 
neoprene commercial glove samples [15] then a year later, the same group measured no penetration for the same gloves with $40 \mathrm{~nm}$ graphite and $10 \mathrm{~nm} \mathrm{TiO}{ }_{2}$ and $\mathrm{Pt}$ particles (Golanski et al. 2009). In 2010, Park et al. studied the penetration of silver nanoaerosols (AgNP) through nitrile rubber and latex gloves using the same experimental setup than Golanski (Park et al., 2011). The authors have concluded to a non-penetration of AgNP through latex and nitrile rubber protective gloves. All these results seem conflicting.

Exposure to nanoparticles in occupational settings may also involve solutions. This situation is especially relevant to protective gloves. Vinches et al. exposed nitrile glove samples to $\mathrm{nTiO}_{2}$ solution in water with dynamic deformations simulating flexing $[16,17]$. ICPMS analyses of sampling solutions suggest the penetration of the nitrile gloves when they were subjected to dynamic mechanical deformations for periods of 5 hours or more.

This paper evaluates the efficiency of four common models of protective gloves against $\mathrm{nTiO}_{2}$ in solutions or in powder in conditions simulating occupational use.

\section{Materials}

\section{1. Protective Gloves}

Four models of protective gloves corresponding to three types of elastomers were selected for this study: disposable latex gloves $(100 \mu \mathrm{m}$ thick - Microflex, Reno, $\mathrm{NV}$ ), disposable nitrile rubber gloves (100 and $200 \mu \mathrm{m}$ thick - Showa Best Glove, Coaticook, QC) respectively identified as NBR-100 and NBR-200, and butyl rubber gloves were also used ( $350 \mu \mathrm{m}$ thick - Showa Best Glove, Coaticook, QC). All the samples were taken from back and the palm section of the gloves.

\section{2. Nanoparticles}

$\mathrm{nTiO}_{2}$ is labelled as $99.7 \%$ pure anatase with an average particle size of $15 \mathrm{~nm}$. Two types of $\mathrm{nTiO}_{2}$ solutions were employed: in water (15 wt\%, Nanostructured \& Amorphous Materials, Inc., Houston, TX) and in 1,2-propanediol, PG (20 wt\%, MK Impex, Mississauga, ON). $\mathrm{nTiO}_{2}$ in powder were also used (Nanostructured \& Amorphous Materials, Inc., Houston, TX).

\section{Methods}

\section{1. Characterization of the $\mathrm{nTiO}_{2}$}

A series of experiments were performed to characterize the nanoparticles solution. Firstly, analysis of the $\mathrm{nTiO}_{2}$ native stock solution (following dilution to
$10 \mathrm{mg} \mathrm{\textrm {L } ^ { - 1 }}$ ) by fluorescence correlation spectroscopy (FCS) gave a hydrodynamic diameter $[18,19]$. Secondly, thermogravimetric analysis (TGA, Diamond TGA/DTA Perkin Elmer) was used to evaluate the mass ratio of the $n \mathrm{TiO}_{2}$ and to identify the presence of chemicals in the colloidal solutions, in addition to the liquid carrier. Gradual evaporation of the liquid carrier occurred between 25 and $150{ }^{\circ} \mathrm{C}$ with a step of $5^{\circ} \mathrm{C} / \mathrm{min}$ [19]. Finally, similar comparisons were performed for the technical grade and ultra-high purity solvents and the $\mathrm{nTiO}_{2}$ suspensions using Fourier transform infrared spectroscopy (FT-IR, Nicolet Continuum XL). Measurements were made in attenuated total reflectance (ATR) mode, between 500 and $4000 \mathrm{~cm}^{-1}$, on drops of solutions after almost total evaporation of the solvent [19]. To obtain statistically significant data, triplicate measurements were performed for all tests.

\section{2. Characterization of the Protective Gloves}

The surface morphology of five samples for each elastomer was analysed by scanning electron microscopy (SEM, Hitachi S3600N - Vacc $=15 \mathrm{kV}-$ magnification $\times 1000$ ) (Vinches et al. 2013). A gold layer is deposited by metallization on the samples. The conductive layer obtained with a vacuum metallizer has a controlled uniform thickness $(15 \mathrm{~nm})$ over the entire sample's surface. Some characteristic features can be observed on the gloves surface: micrometer-size pores for nitrile rubber, cracks for latex and platelets for butyl rubber gloves. The quantification of the surface area of these features was performed using the software ImageJ (image processing).

In the same time, a XPS (X-ray photoelectron spectrometry) modulus is employed to perform chemical analyses of the glove materials, particularly to evaluate the presence or absence of titanium dioxide, used as reinforcing fillers (Mellstrom and Bowan 2005).

\section{3. Mechanical Deformations Experimental Setup}

For the purpose of this study, a test setup has been developed and is illustrated in Fig. 1. It includes an exposure chamber and a sampling chamber, which are separated by the sample. Both chambers and all elements in contact with NP are made of ultrahigh molecular weight polyethylene to limit the effect of adsorption of $\mathrm{nTiO}_{2}$ in solution. The setup has been designed to allow exposing glove samples to both NP when simultaneously subjecting them to dynamic mechanical constraints. $\mathrm{nTiO}_{2}$ are introduced $(6 \mathrm{~mL}$ $n \mathrm{TiO}_{2}$ solution and $250 \mathrm{mg}$ for $\mathrm{nTiO}_{2}$ in powder) in the 
exposure chamber and put in contact with the external surface of the glove samples. As shown in Fig. 1, the test setup is also equipped with a probe linked to an electronic system for controlling mechanical deformations to the sample. The system is computer controlled and includes a 200-N load cell and a position detector. The whole system is enclosed in a glove box to ensure the operator safety during assembly, dismounting and clean-up operations as well as during the tests.

The time profile of sample deformations corresponding to the results reported in this paper is a $50 \%$ deformation every minute. The maximum of BD is fixed to 180 for NBR-100, NBR-200 and latex gloves. In fact, this value corresponds approximately to 3-hours wearing time. Normally, after this time these gloves models should be discarded. Concerning butyl rubber gloves (non-disposable gloves) the maximum of $\mathrm{BD}$ is fixed to 420 (7-hours wearing time).

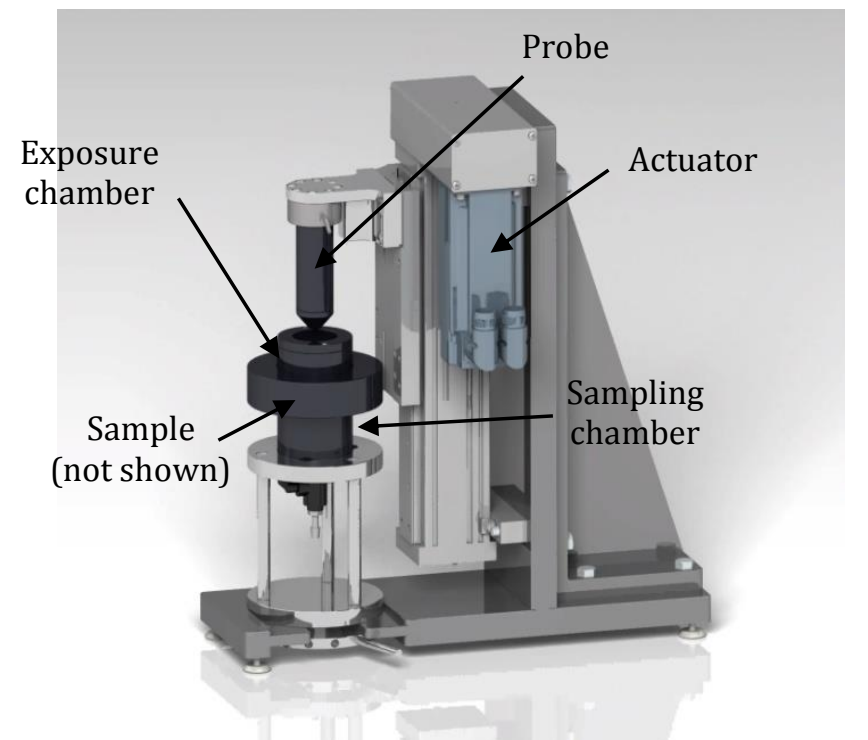

Figure 1. Isometric view of the test setup

\section{4. ICP-MS Analysis}

The preparation of the sampling solutions for ICPMS (PerkinElmer NexION 300X) analyzes was performed following a protocol established by Shaw et al. [20]. Firstly, to minimize adsorption phenomena, aggregation and agglomeration, all sample solutions were stirred vigorously and placed in an ultrasonic bath (Branson model 5510) for 20 minutes. Secondly, $4 \mathrm{~mL}$ of sampling solution is acidified to $2 \%$ with the addition of $150 \mu \mathrm{L}$ of pure nitric acid (Fluka Analytical , $\mathrm{HNO}_{3} \geq 65 \%$, the presence of $\mathrm{Ti} \leq 0.01 \mathrm{mg} / \mathrm{kg}$ ). Finally, $1 \mathrm{~mL}$ of a $10 \%$ -
Triton X-100 is added. Triton X-100 is a non-ionic surfactant in liquid form. According to Shaw, Triton X100 facilitates the $\mathrm{nTiO}_{2}$ dispersion in the matrix. Before being analyzed by ICP- MS, each sample was stirred during 10 seconds.

ICP-MS does not measure directly $\mathrm{nTiO}_{2}$ concentration $\left(\left[\mathrm{nTiO}_{2}\right]\right)$ but the concentration of $\mathrm{Ti}^{4+}$ ([ $\left.\left.\mathrm{Ti}^{4+}\right]\right)$. Indeed, after the introduction of the sampling solution, $\mathrm{nTiO}_{2}$ are ionized according to Equation (1) that is corresponding in molar concentration terms at the Equation (2).

$$
\begin{aligned}
& \mathrm{nTiO}_{2} \rightarrow \mathrm{Ti}^{4+}+2 \mathrm{O}^{2-} \\
& {\left[\mathrm{TiO}_{2}\right]=\left[\mathrm{Ti}^{4+}\right]=2\left[\mathrm{O}^{2-}\right]}
\end{aligned}
$$

ICP-MS give mass concentration expressed in $\mu \mathrm{g} / \mathrm{L}$. To convert molar concentration to mass concentration, we must multiply the molar concentration by the molar mass of chemical compound as Equation (3):

$$
\left[\mathrm{TiO}_{2}\right] \times \mathrm{M}_{\mathrm{TiO}_{2}}=\left[\mathrm{Ti}^{4+}\right] \times \mathrm{M}_{\mathrm{Ti}^{4+}}
$$

With $M_{\mathrm{TiO}_{2}}=79.866 \mathrm{~g} / \mathrm{mol}$ and $M_{T i}=47.867 \mathrm{~g} /$ $\mathrm{mol}$. So, a proportional relationship can be established between the mass concentrations of $\mathrm{nTiO}_{2}$ and $\mathrm{Ti}^{4+}$ (Equation (4)):

$$
\left[\mathrm{TiO}_{2}\right]=1.668 \times\left[\mathrm{Ti}^{4+}\right]
$$

In addition, titanium has five naturally occurring isotopes. ICP-MS measure simultaneously the concentrations of four of them: ${ }^{46} \mathrm{Ti},{ }^{47} \mathrm{Ti},{ }^{48} \mathrm{Ti},{ }^{49} \mathrm{Ti}$. To minimize interference with other chemical groups, for example $S-H$ group, only the concentrations of ${ }^{47} \mathrm{Ti}$ are retained.

\section{Result and Discussion}

\section{1. Characterization of the $\mathrm{nTiO}_{2}$ Solutions}

FCS analyses were performed to measure the hydrodynamic diameter of the $\mathrm{nTiO}_{2}$ solutions. $21 \pm 2 \mathrm{~nm}$ were obtained for $\mathrm{nTiO}_{2}$ in water [19]. The same analysis was not possible for the $\mathrm{nTiO}_{2}$ in $\mathrm{PG}$ due to an incompatibility between the cell material and the colloidal solution liquid carrier. Thermogravimetric analyses were also carried out to estimate the $\mathrm{nTiO}_{2}$ mass fractions. The mass fraction obtained for $\mathrm{nTiO}_{2}$ in water is $14.3 \pm 0.8 \%$ and $25.0 \pm 3.7 \%$ for $\mathrm{nTiO}_{2}$ in PG 
[19]. These results confirmed the manufacturer data. In addition, FT-IR analyses were performed to bring out the presence of additives such as stabilizing agents. An additional peak appears at $1070 \mathrm{~cm}^{-1}$ for the $\mathrm{nTiO}_{2}$ in water (no shown) compared to the spectrum of milli- $Q$ water [19]. This peak may be associated with the elongation of a $\mathrm{CO}$ bond. It might indicate the presence of an alcohol or ether used as additive in the $\mathrm{nTiO}_{2}$ solution. On the other hand, the spectra of $\mathrm{nTiO}_{2}$ in PG and the ultra pure PG do not disclose any significant difference. In these cases, spectra exhibit a complex structure which explains the difficulty in detecting additional peaks associated with the presence of additives.

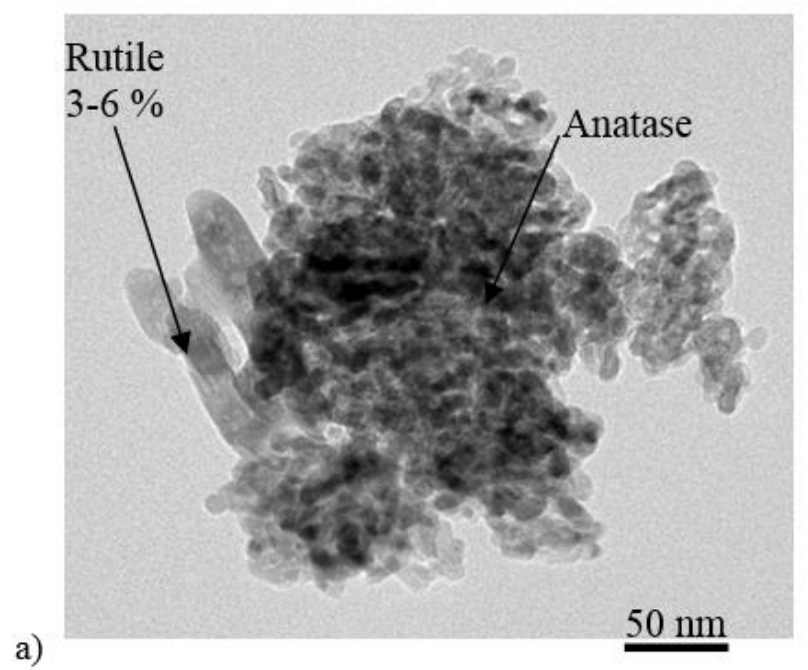

\section{2. Characterization of the $\mathrm{nTiO}_{2}$ Powder}

The size distribution of the $\mathrm{nTiO}_{2}$ was measured by transmission electron microscopy (TEM, JEM-2100F) and verified by statistical analysis of 174 particles. Two allotropic forms of $\mathrm{TiO}_{2}$ were observed: a spherical anatase and a rod-like rutile (Fig 2 (a)). Analysis by X-ray diffraction (Philips X'PERT) confirmed the presence of 3 to $6 \%$ rutile in the $\mathrm{nTiO}_{2}$. Fig 2(b) displays the size distribution of the analysed sample in terms of circular diameter. It can be seen that the average dimension of the particles/aggregates was situated around $100 \mathrm{~nm}$, although even some micrometric-size agglomerates were recorded. In fact, only two particles with a diameter lower than $20 \mathrm{~nm}$ were counted.

b)

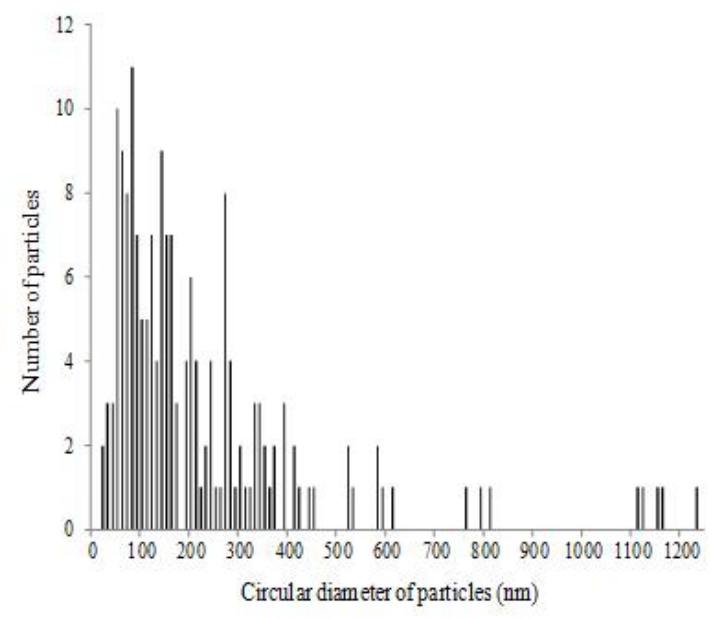

Figure 2. (a) TEM image of the $\mathrm{nTiO}_{2}$ powder and (b) Size distribution of the $\mathrm{nTiO}_{2}$ powder

\subsection{Characterization of the Protective Gloves Surface}

Micrometer-size surface features which can be observed on the outer surface (surface in contact with the $\mathrm{nTiO}_{2}$ ) of all the protective gloves (Figure 3). For both nitrile rubber gloves, the surface features corresponding to pores, cracks for latex glove and platelets for butyl rubber gloves. These surface features may facilitate the penetration of $\mathrm{nTiO}_{2}$ through protective gloves [21].
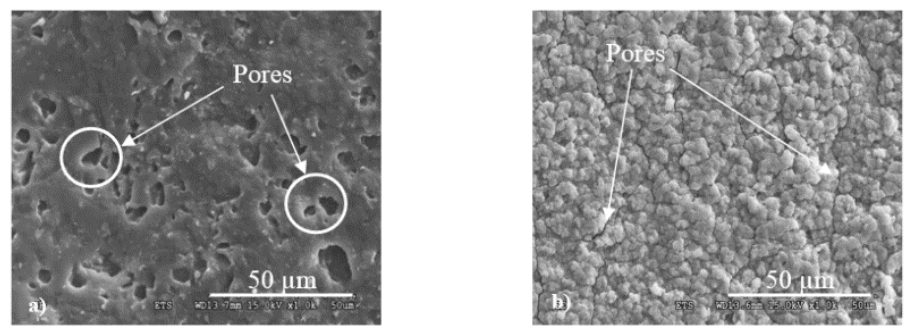

Figure 3. SEM images of the native outer surfaces of protective gloves (a) NBR-100 and (b) latex
XPS chemical analyses of the glove material have been made. Triplicate measurements were performed for all analyses. The mass concentrations of titanium measured are $(0.44 \pm 0.08)$ for NBR-100 and $(0.73 \pm$ $0.14)$. No trace of titanium is detected for latex and butyl rubber gloves. To ensure that the titanium concentration in the sampling chamber is due to the passage of $\mathrm{nTiO}_{2}$ and not to the glove degradation, some tests were performed without NP.

\section{4. ICP-MS Results}

The variation of $\mathrm{Ti}^{4+}$ concentration in the sampling solutions as a function of the number of deformations is reported in Figure 4 and corresponding to NBR-100 samples in contact or not with $\mathrm{nTiO}_{2}$ solutions. To obtain statistically significant data, triplicate measurements were performed for all analyses. Initial tests were performed without $\mathrm{nTiO}_{2}$ to determine the possible 
contamination by titanium content in nitrile rubber gloves. The average value of $\mathrm{Ti}^{4+}$ concentration is 0.4 $\mu \mathrm{g} / \mathrm{L}$ (dashed line in Figure 4). Similarly, in the presence of $\mathrm{nTiO}_{2}$ in $\mathrm{PG}$, no penetration of NP is remarkable. This result indicates that NBR-100 is efficient against the penetration $\mathrm{nTiO}_{2}$ in $\mathrm{PG}$ under work conditions. By cons, for $\mathrm{nTiO}_{2}$ in water, the maximum value of $\mathrm{Ti}^{4+}$ concentration is reached for $180 \mathrm{DB}(2590 \mu \mathrm{g} / \mathrm{L})$. According to the Equation (4), $\mathrm{nTiO}_{2}$ concentration is $4320 \mu \mathrm{g} / \mathrm{L}$. It may be noted that $\mathrm{nTiO}_{2}$ concentration is $12.8 \mu \mathrm{g} / \mathrm{L}$ after $60 \mathrm{BD}$ and $271.9 \mu \mathrm{g} / \mathrm{L}$ after $120 \mathrm{BD}$. This result can be explained by a progressive deterioration of these protective gloves. No significant $\mathrm{Ti}^{4+}$ concentration was measured for NBR-200, latex and butyl gloves in contact with $\mathrm{nTiO}_{2}$ in solutions.

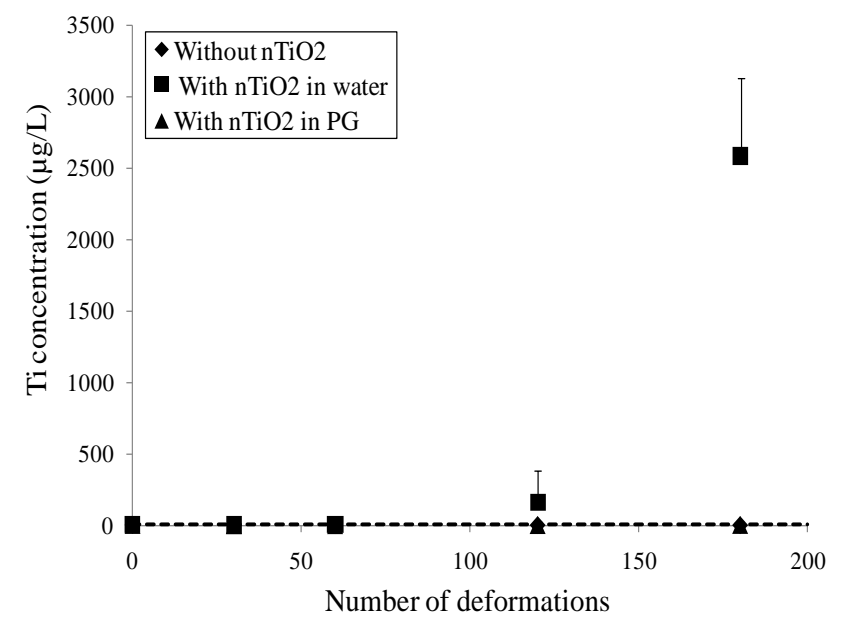

Figure 4. Titanium concentration in the sampling solutions as a function of the number of deformations for NBR-100 samples exposed to $\mathrm{nTiO}_{2}$ solutions

Same tests were performed with $\mathrm{nTiO}_{2}$ in powder. In this case, no major $\mathrm{Ti}^{4+}$ concentration was measured for NBR-200 and latex gloves. By cons, for butyl rubber, $\mathrm{Ti}^{4+}$ concentration reached $10.9 \mu \mathrm{g} / \mathrm{L}$ after $420 \mathrm{BD}$ (Figure 5). Similarly, a significant increase in $\mathrm{Ti}^{4+}$ concentration was detected with NBR-100. However, these results should be confirmed because the protocol established by Shaw et al. [20] gives a yield of $45 \%$ with $\mathrm{nTiO}_{2}$ in powder whereas it reaches $85 \%$ with $\mathrm{nTiO}_{2}$ in solutions.

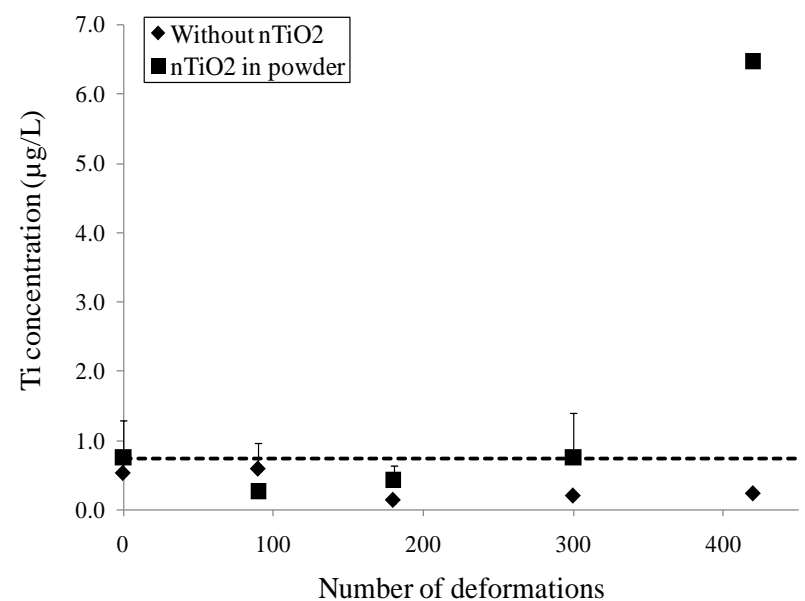

Figure 5. Variation in titanium concentration in the sampling solutions as a function of the number of deformations for butyl rubber samples exposed to $\mathrm{nTiO}_{2}$ in powder

\section{Conclusion}

This work has experimentally evaluated the efficiency of protective gloves against the $\mathrm{nTiO}_{2}$ under work conditions. The results are different depending on the glove models and the $\mathrm{nTiO}_{2}$ application mode. Table 1 summarizes the efficiency of all the protective gloves studied against $\mathrm{nTiO}_{2}$.

Table 1. Efficiency of protective gloves

\begin{tabular}{c|cccc}
\hline & NBR-100 & $\begin{array}{c}\text { NBR- } \\
200\end{array}$ & Latex & $\begin{array}{c}\text { Butyl } \\
\text { rubber }\end{array}$ \\
\hline $\begin{array}{c}\mathrm{nTiO}_{2} \text { in } \\
\text { water } \\
\mathrm{nTiO}_{2} \text { in } \\
\mathrm{PG}\end{array}$ & Poor & Good & Good & Good \\
$\begin{array}{c}\mathrm{nTiO}_{2} \text { in } \\
\text { powder }\end{array}$ & Weak & Good & Good & Good \\
\hline
\end{tabular}

In the case of NBR-200, the thickness has a major role as barrier whereas for latex gloves, the chemical composition seems to be the main actor in the efficiency. Some tests are needed to confirm the results obtained with $\mathrm{nTiO}_{2}$ in powder. So we need for further investigations but for the moment, great care must be taken in selecting protective gloves for the handling $n \mathrm{TiO}_{2}$. It is already possible to recommend a frequent replacement of gloves in case of exposure to $\mathrm{nTiO}_{2}$.

\section{Acknowledgements}

The authors would like to acknowledge the contribution of M. Ben Salah (École de technologie supérieure) and Madjid Hadioui (Université de Montréal) to the project. 


\section{References}

[1] Bello, D., et al., Physicochemical and morphological characterisation of nanoparticles from photocopiers: implications for environmental health. Nanotoxicology, 2012. 7(5): p. 989-1003.

[2] Philbert, M.A., et al., Review of Federal Strategy for Nanotechnology-Related Environmental, Health, and Safety Research. 2009: The National Academies Press.

[3] Maynard, A., Nanotechnology and occupational health. 2005, EPA, June 13th 2005.

[4] Roco, M.C., C.A. Mirkin, and M.C. Hersam, Nanotechnology Research Directions for Societal Needs in 2020 - Retrospective and Outlook, S.P. Reports, Editor. 2010, WTEC Study on Nanotechnology Research Directions: Berlin and Boston. p. 610.

[5] Hervé-Bazin, B., Les nanoparticules: Un enjeu majeur pour la santé au travail ? 2007: EDP Sciences. 704.

[6] Robichaud, C.O., et al., Estimates of upper bounds and trends in nano-TiO2 production as a basis for exposure assessment. Environmental Science and Technology, 2009. 43: p. 4227-4233.

[7] IARC, Monographs on the evaluation of carcinogenic risks to humans - carbon black, titanium dioxide and talc. 2010, World health organization: Lyon.

[8] CCHST. Basic Information on Titanium Dioxide. 2007; Available

from: http://www.cchst.ca/oshanswers/chemicals/che m_profiles/titanium_dioxide/basic_td.html.

[9] $\mathrm{Wu}, \mathrm{J}$. , et al., Toxicity and penetration of $\mathrm{TiO} 2$ nanoparticles in hairless mice and porcine skin after subchronic dermal exposure. Toxicology Letters, 2009. 191(1): p. 1-8.

[10] Jang, Y.S., et al., The potential for skin irritation, phototoxicity, and sensitization of $\mathrm{ZnO}$ nanoparticles. Molecular \& Cellular Toxicology, 2012. 8(2): p. 171-177.

[11] Labouta, H.I., et al., Mechanism and determinants of nanoparticle penetration through human skin. Nanoscale, 2011. 3(12): p. 4989-4999.

[12] Larese, F.F., et al., Human skin penetration of silver nanoparticles through intact and damaged skin. Toxicology, 2009. 255(1-2): p. 33-37.

[13] Ostiguy, C., et al., Les nanoparticules de synthèse Connaissances actuelles sur les risques et les mesures de prévention en SST - 2e édition. 2009, Institut de recherche Robert-Sauvé en santé et en sécurité au travail p. 159.
[14] OECD, Current developments/activities on the safety of manufactured nanomaterials - Tour de table at the 7 th meeting of the working party on manufactured nanomaterials., in Series on the safety of manufactured nanomaterials. 2010: Paris.

[15] Golanski, L., A. Guiot, and F. Tardif. Are conventional protective devices such as fibrous filter media, respirator cartridges, protective clothing and gloves also efficient for nanoaerosols ? 2008; Available from:

http://www.nanosafe.org/home/liblocal/docs/D issemination\%20report/DR1_s.pdf.

[16] Vinches, L., et al., Experimental evaluation of the resistance of protective gloves against titanium dioxide nanoparticles in solution under conditions simulating occupationnal use, in NanoSafe 2012. 2012: Grenoble, France.

[17] Vinches, L., et al., Experimental evaluation of the penetration of TiO2 nanoparticles through protective clothing and gloves under conditions simulating occupational use. Nanoscience Methods, 2013: p. 1-15.

[18] Domingos, R., et al., Characterizing manufactured nanoparticles in the environment: Multimethod determination of particle sizes. Environmental Science and Technology 2009. 43(19): p. 72777284

[19] Vinches, L., et al., Swelling of Protective Gloves in Commercial TiO2 Nanoparticles Colloidal Solutions. International Journal of Theoretical and Applied Nanotechnology, 2012. 1(1): p. 45-51.

[20] Shaw, B.J., et al., A simplified method for determining titanium from TiO2 nanoparticles in fish tissue with a concomitant multi-element analysis. Chemosphere, 2013. 92(9): p. 1136-1144.

[21] Vinches, L., et al., Physical phenomena facilitating the penetration of solutions of $\mathrm{TiO} 2$ nanoparticles through protective gloves. International Journal of Theoretical and Applied Nanotechnology, 2013. 1(2): p. 1-16. 